\title{
Stat-3 is required for pulmonary homeostasis during hyperoxia
}

\author{
Isamu Hokuto, ${ }^{1}$ Machiko Ikegami, ${ }^{1}$ Mitsuhiro Yoshida, ${ }^{2}$ Kiyoshi Takeda, ${ }^{3}$ Shizuo Akira, ${ }^{3}$ \\ Anne-Karina T. Perl, ${ }^{1}$ William M. Hull, ${ }^{1}$ Susan E. Wert, ${ }^{1}$ and Jeffrey A. Whitsett ${ }^{1}$ \\ ${ }^{1}$ Division of Pulmonary Biology, Cincinnati Children's Hospital Medical Center, Cincinnati, Ohio, USA \\ ${ }^{2}$ Department of Molecular Medicine, and \\ ${ }^{3}$ Department of Host Defense, Research Institute for Microbial Diseases, Osaka University Graduate School of Medicine, \\ Suita City, Osaka, Japan
}

\begin{abstract}
Acute lung injury syndromes remain common causes of morbidity and mortality in adults and children. Cellular and physiologic mechanisms maintaining pulmonary homeostasis during lung injury remain poorly understood. In the present study, the Stat-3 gene was selectively deleted in respiratory epithelial cells by conditional expression of Cre-recombinase under control of the surfactant protein $\mathrm{C}$ gene promoter. Cell-selective deletion of Stat-3 in respiratory epithelial cells did not alter prenatal lung morphogenesis or postnatal lung function. However, exposure of adult Stat-3-deleted mice to $95 \%$ oxygen caused a more rapidly progressive lung injury associated with alveolar capillary leak and acute respiratory distress. Epithelial cell injury and inflammatory responses were increased in the Stat-3-deleted mice. Surfactant proteins and lipids were decreased or absent in alveolar lavage material. Intratracheal treatment with exogenous surfactant protein B improved survival and lung histology in Stat-3-deleted mice during hyperoxia. Expression of Stat-3 in respiratory epithelial cells is not required for lung formation, but plays a critical role in maintenance of surfactant homeostasis and lung function during oxygen injury.
\end{abstract}

J. Clin. Invest. 113:28-37 (2004). doi:10.1172/JCI200419491.

\section{Introduction}

Signal transducers and activators of transcription (STATs) comprise a family of seven related polypeptides that participate in signaling pathways mediating a wide variety of cellular and organ responses to cytokines and growth factors. While specific STATs generally mediate signals provided by relatively specific receptors, STAT-3 participates in numerous pathways from a large number of diverse growth factors and cytokines. STAT-3 was initially identified as a nuclear factor mediating IL-6-dependent signaling involved in acute-phase responses in the liver. Subsequent studies demonstrated that STAT-3 mediates or participates in the signaling from many cytokines (e.g., IL-6, IL-11, IL-10,

Received for publication July 15, 2003, and accepted in revised form November 4, 2003.

Address correspondence to: Jeffrey A. Whitsett, Cincinnati Children's Hospital Medical Center, Divisions of Neonatology and Pulmonary Biology, 3333 Burnet Avenue, Cincinnati, Ohio 45229-3039, USA. Phone: (513) 636-4830; Fax: (513) 636-7868;

E-mail: jeff.whitsett@cchmc.org.

Conflict of interest: The authors have declared that no conflict of interest exists.

Nonstandard abbreviations used: signal transducer and activator of transcription (STAT); Janus kinase (JAK); surfactant protein B (SP-B); acute lung injury (ALI); acute respiratory distress syndrome (ARDS); severe acute respiratory syndrome (SARS); SP-C pro-protein (proSP-C); conditional KO (Stat-3 ${ }^{\Delta / \Delta}$ ); bronchoalveolar lavage fluid (BALF); saturated phosphatidylcholine (SatPC); macrophage inflammatory protein-2 (MIP-2); DL- $\alpha$ dipalmitoyl phosphatidylcholine/ 1-palmitoyl-2-oleoyl-sn-glycero-3-[phospho-rac-(1-glycerol)] (DPPC/POPG); respiratory distress syndrome (RDS).
IL-2, leukemic inhibitory factor, ciliary neutrotropic factor, oncostatin $M$, and others), as well as growth factors (e.g., EGF, TGF- $\alpha$, hepatocyte growth factor, leptin, G-CSF) in various cells and organs (reviewed in refs. $1-3)$. STAT-3 is activated by phosphorylation by Janus kinases (JAKs). Phosphorylation mediates dimerization, nuclear translocation, and the subsequent activation of transcriptional targets. Deletion of the Stat-3 gene in transgenic mice demonstrated that it was essential for embryonic survival near the time of implantation (E6.5-7.5) (4). Therefore, its biological roles have been determined primarily in in vitro experiments and after conditional deletion in various cell types and organs. STAT-3 plays diverse roles in biological processes including cell migration, survival, proliferation, apoptosis, and inflammation. While deletion of Stat-3 caused early embryonic lethality and blocked survival of embryonic stem cells, tissue-specific deletion of the Stat-3 gene has not been lethal in various organs and cells, including skin, mammary glands, liver, thymic epithelium, lymphocytes, macrophages, and neurons (see ref. 5 for review).

STAT-3 is expressed in various cell types in the developing and postnatal lung. While its potential role in lung morphogenesis or function is presently unknown, in vitro studies demonstrated that STAT-3 regulated the expression of surfactant protein B (SP-B), binding and activating cis-active elements in the $S f t p B$ gene promoter (6). Its potential role in the regulation of surfactant homeostasis in respiratory epithelial cells in vivo has not been evaluated, however. 
Acute lung injury (ALI) and acute respiratory distress syndrome (ARDS) remain a common cause of morbidity and mortality in both infants and adults following infection, trauma, inhalation of toxicants, and drowning. The recent severe acute respiratory syndrome (SARS) outbreak emphasizes the severity of pulmonary outcomes associated with ALI (7). ARDS is associated with surfactant deficiency and dysfunction $(8,9)$, including abnormalities in surfactant lipids and proteins. Of the surfactant proteins, SP-B is required for maintenance of lung function in both newborn and postnatal periods $(10,11)$ and for adaptation to lung injury following infection or oxygen exposure. To assess the role of STAT-3 in lung function, we used a conditional system to express Cre-recombinase to selectively delete the Stat-3 gene in bronchiolar and alveolar epithelial cells of the mouse lung.

\section{Methods}

Gene construction and PCR. We generated SP-C-rtTAtg/transgenic mice and (tetO) $)_{7} \mathrm{CMV}-\mathrm{Cr} \mathrm{e}^{\mathrm{tg} / \mathrm{tg}}$ or $(\text { tetO })_{7} \mathrm{CMV}-$ $\mathrm{Cre} \mathrm{rg}^{\mathrm{tg}-}$ mice as described previously $(12,13)$. Stat- $3^{\mathrm{flx} / \mathrm{flx}}$ mice were generated by K. Takeda (14). SP-C-rtTA ${ }^{\mathrm{tg} /}$, or (tetO) $)_{7} \mathrm{CMV}-\mathrm{Cr} \mathrm{e}^{\mathrm{tg} / \mathrm{tg}}$, or (tetO) ${ }_{7} \mathrm{CMV}-\mathrm{Cre}^{\mathrm{tg} /} /$ transgenic $^{-}$ mice were mated with Stat- $3 \mathrm{flx} / \mathrm{flx}$ mice to generate double-transgenic mice that have SP-C-rtTA $A^{\text {tg }}-/$ Stat- $3 f \mid x /+$, or

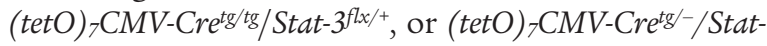
$3^{f x x+}$. Mice were mated to generate SP-C-rtTA/Stat-3ffx/flx or (tetO) ${ }_{7} \mathrm{CMV}$-Cretg/tg/Stat-3flx/flx or (tetO) ${ }_{7} \mathrm{CMV}$-Cretg $/$ - Stat$3^{f l x f f l x}$ mice. These mice were mated to generate SP-C-

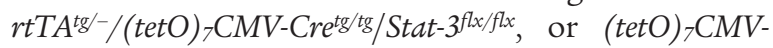
$\mathrm{Cr} e^{\text {tg }} /$ /Stat- $3 f^{f x / f l x}$ triple transgenic mice. To confirm the genotype, DNA was purified from tail or lung of experimental mice and PCR was performed for SP-C-rtTA, (tet $O)_{7} C M V$-Cre and Stat-3 ${ }^{f t x}$ genes using the primers $5^{\prime}$ GAC ACA TAT AAG ACC CTG GCTA- $3^{\prime}$ and $5^{\prime}$-AAA ATC TTG CCA GCT TTC CC- $3^{\prime}$ for SP-C-rtTA; $5^{\prime}$-TGC CAC CAA GTG ACA GCA ATG- $3^{\prime}$ and $5^{\prime}$-AGA GAC GGA AAT CCA TCG CTCG- $3^{\prime}$ for (tetO) ${ }_{7}$ CMV-Cre; and $5^{\prime}$-CCT GAA GAC CAA GTT CAT CTG TGT GAC- $3^{\prime}$ and $5^{\prime}$-CAC ACA AGC CAT CAA ACT CTG GTC TCC-3' for Stat-3fix. Triple transgenic (Stat$3^{\Delta / \Delta}$ ) and nondeleted littermate (Stat-3ffloflx) control mice were used for the experiments.

Animal use and doxycycline administration. Transgenic $S t a t-3^{\Delta / \Delta}$ and control mice were kept in a pathogen-free vivarium according to institutional guidelines until oxygen exposure. Doxycycline was administered to the dams in the food at a concentration of $625 \mathrm{mg} / \mathrm{kg}$ (Harlan Teklad, Madison, Wisconsin, USA) from E0 to P25. Mice were then provided normal food.

Isolation and purification of type II epithelial cells and quantification of STAT-3 $m R N A$. Type II cells were isolated from control and Stat-3 $3^{\Delta / \Delta}$ mice using collagenase and differential plating on Matrigel (15). After 5 days in culture, cells were removed from the Matrigel with Dispase and centrifuged for 5 minutes, the pellet was homogenized in TRIzol reagent (Invitrogen Corp., Carlsbad, California, USA), and the RNA was extracted. After DNase treatment, cDNA was synthesized by using Superscript II.
Quantitative RT-PCR for STAT-3 and $\beta$-actin mRNAs was performed using Smart Cycler (Cepheid, Sunnyvale, California, USA). Primers 5'-GAA GAC CAA GTT CAT CTG TGTG-3' (specific for exon 21) and 5'-GTA GCA CAC TCC GAG GTC AGAT- $3^{\prime}$ for STAT- 3 and $5^{\prime}$-TGG AAT CCT GTG GCA TCC ATG AAC- $3^{\prime}$ and $5^{\prime}$-TAA AAC GCA GCT CAG TAA CAG TCCG- $3^{\prime}$ for $\beta$-actin were used.

Tissue preparation, histology, and immunohistochemistry. Mice were killed by an injection of pentobarbital. Lungs were inflation fixed with $4 \%$ paraformaldehyde in PBS at $25 \mathrm{cmH}_{2} \mathrm{O}$ and immersed in the same fixative. Tissue was fixed overnight, washed with PBS, dehydrated in the series of alcohols, and embedded in paraffin. Tissue sections were stained with H\&E for histology. Immunohistochemistry for STAT-3 was performed on tissue sections using a microwave antigen-retrieval technique (16). Sections were pretreated with $3 \% \mathrm{H}_{2} \mathrm{O}_{2}$ in methanol for inactivation of endogenous peroxidase, then placed in $4 \%$ normal goat serum or Background Buster (Innovex Bioscience, Richmond, California, USA) for 2 hours before incubation with rabbit polyclonal primary antisera for STAT-3 (1:2,000; Santa Cruz Biotechnology, California, USA) in $4 \%$ normal goat serum overnight at $4^{\circ} \mathrm{C}$. After rinsing, tissue sections were incubated with biotinylated anti-rabbit IgG goat $\mathrm{Ab}(7.5 \mu \mathrm{g} / \mathrm{ml}$; Vector Laboratories Inc., Burlingame, California, USA) for 30 minutes and detected with avidin-biotin complex detection kit (Vecstatin Elite ABC kit; Vector Laboratories Inc.) developed with diaminobenzidine. Specimens were counterstained with $0.1 \%$ of nuclear fast red. Immunohistochemistry for SP-B and SP-C pro-protein (proSP-C) was performed as previously described using rabbit polyclonal Ab's (17). Caspase-3 (Biocare Medical, Walnut Creek, California, USA), TUNEL (Trevigen Inc., Gaithersburg, Maryland, USA), and keratin-18 fragment (Roche Molecular Biochemicals, Mannheim, Germany) Ab's were used to assess apoptosis.

Morphometric analysis. Severity of lung injury was assessed using a scoring system that included assessment of alveolar capillary membranes, bronchiolar injury, inflammation, and remodeling. Lung sections from multiple lobes of Stat- $3^{\Delta / \Delta}$ and control mice $(n=6$ per group) were assessed after 65 hours in 95\% oxygen by a blinded reviewer. Nonparametric analysis of the median score for each characteristic was used to determine significant differences.

$R N A$ extraction and $S 1$ nuclease assay of SP-A, SP-B, and $S P$-C $m R N A$. Lung tissues were excised and homogenated with TRIzol reagent (Invitrogen Corp.). RNA was measured by spectrophotometer. S1 nuclease assays for SP-A, SP-B, SP-C, and L32 were performed as previously described (18) and quantified using ImageQuant analysis software.

Hyperoxia and pulmonary mechanics. Stat-3 $3^{\Delta / \Delta}$ and littermate control mice ( $n=5$ per group) were exposed either to $95 \% \mathrm{O}_{2}$ or to room air for 65 hours, the timing based on previous observations regarding the duration of oxygen exposure inducing respiratory distress 
a
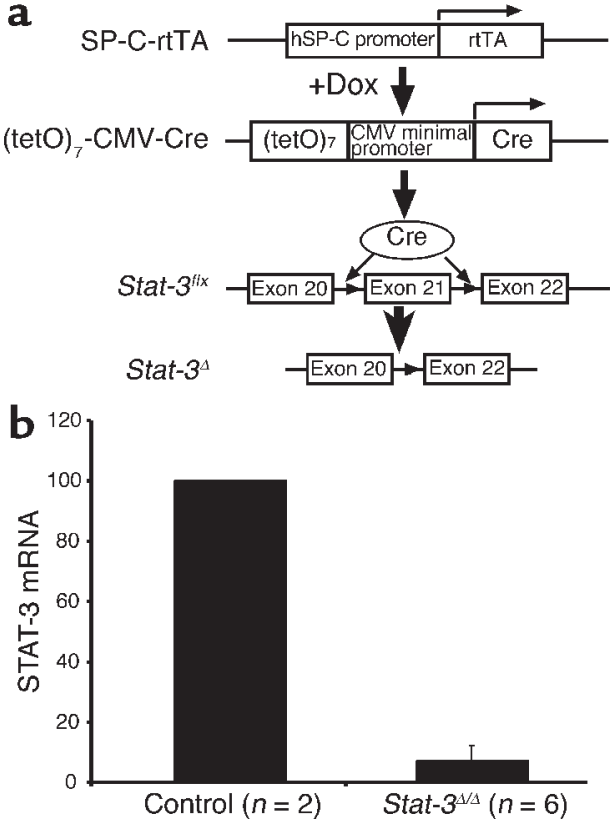

in $95 \%$ oxygen (19). Lung mechanics were studied in tracheostomized mice under anesthesia by intraperitoneal injection of $0.1 \mathrm{ml} / 10 \mathrm{~g}$ body weight of a mixture containing $40 \mathrm{mg} / \mathrm{ml}$ ketamine and $2 \mathrm{mg} / \mathrm{ml}$ xylazine. Mice were ventilated with a tidal volume of $8 \mathrm{ml} / \mathrm{kg}$ at a rate of 450 breaths/min and a positive end-expiratory pressure of $2 \mathrm{cmH}_{2} \mathrm{O}$ by a computerized FlexiVent system (SCIREQ Scientific Respiratory Equipment, Montreal, Quebec, Canada) (20). This machine allows accurate measurement of volume by using the position of the ventilator piston and pressure in the cylinder. After mechanical ventilation for 2 minutes, two measurement maneuvers were performed, as follows. For the first maneuver, a sinusoidal $1 \mathrm{~Hz}$ oscillation was applied to the tracheal tube. The single compartment model was fit to these data using multiple linear regression in order to calculate dynamic resistance, elastance, and compliance of the respiratory system (airway). For the second maneuver, an 8-second forced oscillatory signal containing frequencies between 0.5 and $19.6 \mathrm{~Hz}$ was applied to the tracheal tube. The mechanical input impedance of the respiratory system was calculated, and

\section{Figure 2}

STAT-3 immunostaining in the Stat- $3^{\Delta / \Delta}$ mice. Dams were treated with doxycycline from E0. Lung sections were prepared at E18.5 (a-d) or at 6 weeks of age (e-h). Tissues were stained with H\&E (a, $\mathbf{c}, \mathbf{e}, \mathbf{g})$ or immunostained for STAT-3 (b, d, f, h). Lung morphology was not altered in the Stat- $3^{\Delta / \Delta}$ mice. Complete or nearly complete loss of STAT-3 staining was observed in respiratory epithelial cells at E18. Likewise, staining was observed in alveolar type II and conducting airway cells at PN 6 weeks in Stat- $3^{\Delta / \Delta}$ mice (arrows). STAT-3 was detected in alveolar macrophages and in other nonepithelial cells in control and Stat-3 $3^{\Delta / \Delta}$ mice ( $\mathbf{f}$ and $\mathbf{h}$ ) (arrowheads). Figures are representative of $n \geq 3$ per genotype. Scale bar: $100 \mu \mathrm{m}(\mathbf{a}, \mathbf{c}, \mathbf{e}$, g) or $50 \mu \mathrm{m}(\mathbf{b}, \mathbf{d}, \mathbf{f}, \mathbf{h})$.

\section{Figure 1}

Conditional deletion of Stat-3 in respiratory epithelium. (a) The human SP-C (hSP-C) promoter was used to express the reverse tetracycline transactivator (rtTA). In the presence of doxycycline (Dox), rtTA binds to the (tetO) 7 promoter, activating transcription of Crerecombinase. The loxP sites were inserted in introns 20 and 21 of the Stat-3 gene (Stat- $\left.3^{\mathrm{fl} x}\right)$, deleting exon 21 after recombination, to produce the Stat-3 $3^{\Delta}$ locus. (b) STAT-3 RNA was assessed in purified alveolar type II cells. RNA was extracted from primary cultures of alveolar type II cells isolated from Stat-3 $3^{\Delta / \Delta}$ and control mice. RNA from control $(n=2)$ or Stat- $3^{\Delta / \Delta}(n=2$ or 4$)$ were pooled and analyzed by real-time RT-PCR. Data were standardized to $\beta$-actin RNA and normalized to control. In the Stat-3 $3^{\Delta / \Delta}$ cells, STAT- 3 mRNA was less than $10 \%$ of control values.

a model containing a constant phase tissue compartment (21) was fit to input impedance in order to evaluate tissue damping, tissue elastance, tissue hysteresivity, and newtonian resistance.

Tissue analysis. Mice were given pentobarbital sodium (100 mg/kg intraperitoneally) and killed by exsanguination. Five $1-\mathrm{ml}$ aliquots of $0.9 \% \mathrm{NaCl}$ were flushed into the lungs, withdrawn by syringe three times for each aliquot, and pooled. Total volume of bronchoalveolar lavage fluid (BALF) was recorded, which was similar to $4.43 \pm 0.05 \mathrm{ml}(n=10)$. The lavaged lung tissue was
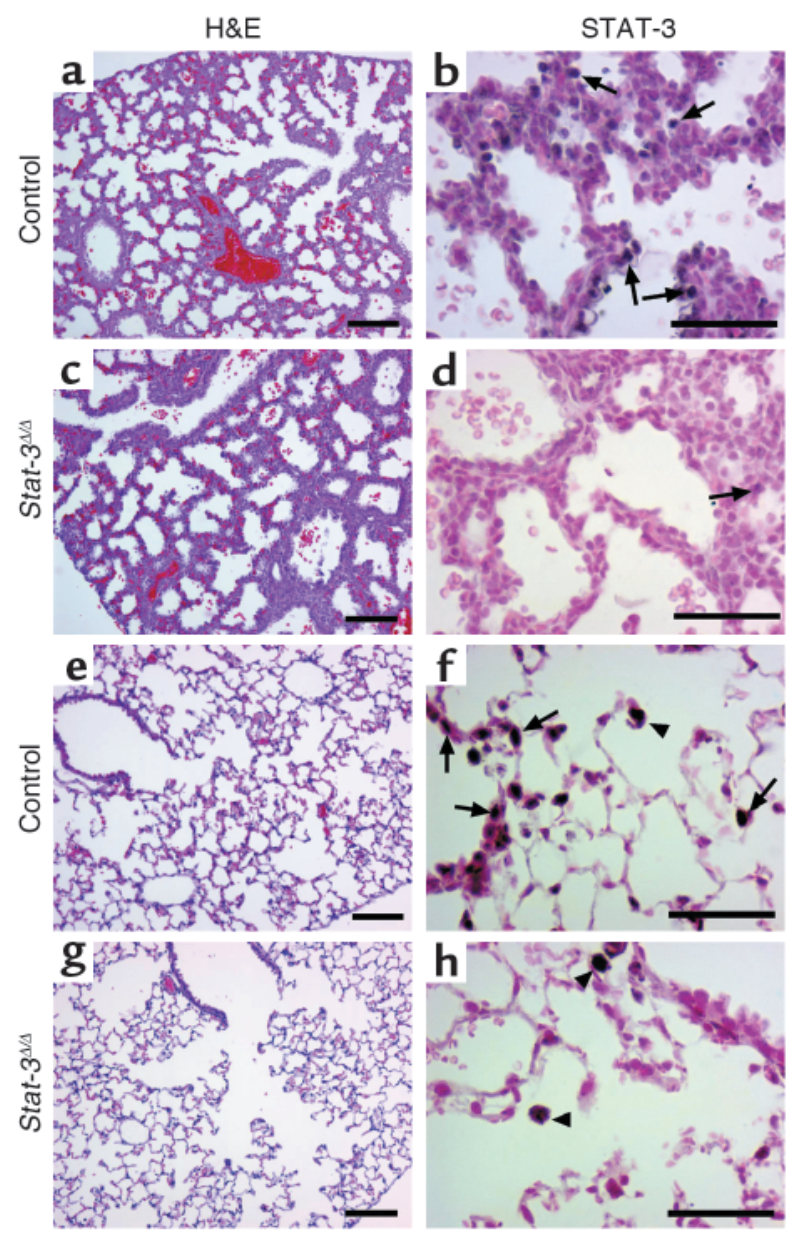


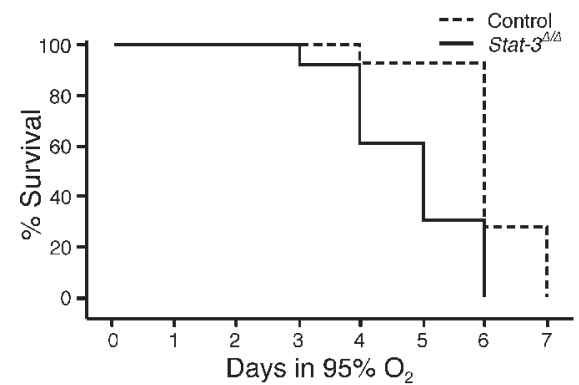

Figure 3

Kaplan-Meier plot of survival of Stat-3 $3^{\Delta / \Delta}$ and control mice during hyperoxia. Adult Stat- $3^{\Delta / \Delta}$ mice and littermates were exposed to $95 \%$ $\mathrm{O}_{2}$. Survival of Stat- $3^{\Delta / \Delta}$ mice in hyperoxia $(n=13)$ was significantly decreased compared with littermate, nondeleted controls $(n=14)$. $P<0.001$ by log-rank test.

removed and homogenized in $2 \mathrm{ml}$ of $0.9 \% \mathrm{NaCl}$. Total protein in aliquots of BALF from each mouse was measured by the method of Lowry et al. (22), and protein in whole BALF was calculated as milligrams per kilogram of body weight. Aliquots of BALF were extracted with chloroform/methanol (2:1), and saturated phosphatidylcholine (SatPC) was isolated with osmium tetroxide (23) followed by measurement of phosphorus (24).

The content of SP-A, SP-B, SP-C, and SP-D in BALF was determined by Western blot analysis. Samples containing $1.25 \mathrm{nmol}$ of SatPC were used for SP-A and SP-D, $0.22 \mathrm{nmol}$ of SatPC was used for analysis of SP-B, and $0.65 \mathrm{nmol}$ of SatPC was used for SP-C. Separation was carried out under nonreducing conditions for SP-B and SP-C and under reducing conditions for SP-A and SP-B. The following dilutions of antisera were used: 1:5,000 for guinea pig anti-rat SP-A, 1:15,000 for rabbit anti-bovine SP-B, 1:10,000 for rabbit anti-recombinant human proSP-C, and 1:5,000 for rabbit anti-rat SP-D (Chemicon International, Temecula, California, USA). Appropriate peroxidase-conjugated secondary Ab's were used at 1:10,000 dilution. Immunoreactive bands were detected with ECL reagents (Amersham Health, Chicago, Illinois, USA). Protein bands were quantitated by densitometric analyses with Alpha Imager 2000 documentation and ImageQuant analyses software. Lung homogenates were centrifuged at $1,500 \mathrm{~g}$, and the supernatants were stored at $-20^{\circ} \mathrm{C}$. IL- $1 \beta$, IL-6, and macrophage inflammatory protein-2 (MIP-2) were quantitated in supernatants using quantitative murine sandwich ELISA kits (R\&D Systems Inc., Minneapolis, Minnesota, USA).

Treatment of Stat- $3^{\Delta / \Delta}$ mice with SP-B during hyperoxia exposure. SP-B was isolated and purified from chloroform/methanol extracts of BALF from a patient with pulmonary alveolar proteinosis using Sephadex LH 60 column as previously described (19) (gift from Timothy E. Weaver, Cincinnati Children's Hospital Medical Center, Cincinnati, Ohio, USA). Purified human SP-B was added to the DL- $\alpha$ dipalmitoyl phosphatidylcholine/1-palmitoyl-2-oleoyl-sn-glycero-3-[phosphorac-(1-glycerol)] (DPPC/POPG), 7:3 by weight (Avanti Polar Lipids Inc., Alabaster, Alabama, USA), to a final concentration of $4 \%$ by weight. The lipid mixture, without SP-B, was given to the comparison group. The lipid mixtures, with and without SP-B, were dissolved in chloroform, dried with $\mathrm{N}_{2}$, and resuspended in sterile $0.9 \% \mathrm{NaCl}$ by sonication. Stat $-3^{\Delta / \Delta}$ mice ( 7 weeks of age) were exposed to $95 \% \mathrm{O}_{2}$ and treated intratracheally with the surfactant mixture 16 and 40 hours after being placed in hyperoxia. Stat-3 $3^{\Delta / \Delta}$ mice were anesthetized by isoflurane inhalation with $95 \% \mathrm{O}_{2}$ and orally intubated with a 24-gauge animal feeding needle, receiving $80 \mu \mathrm{l}$ of $50 \mathrm{mg}$ phospholipids/kilogram of
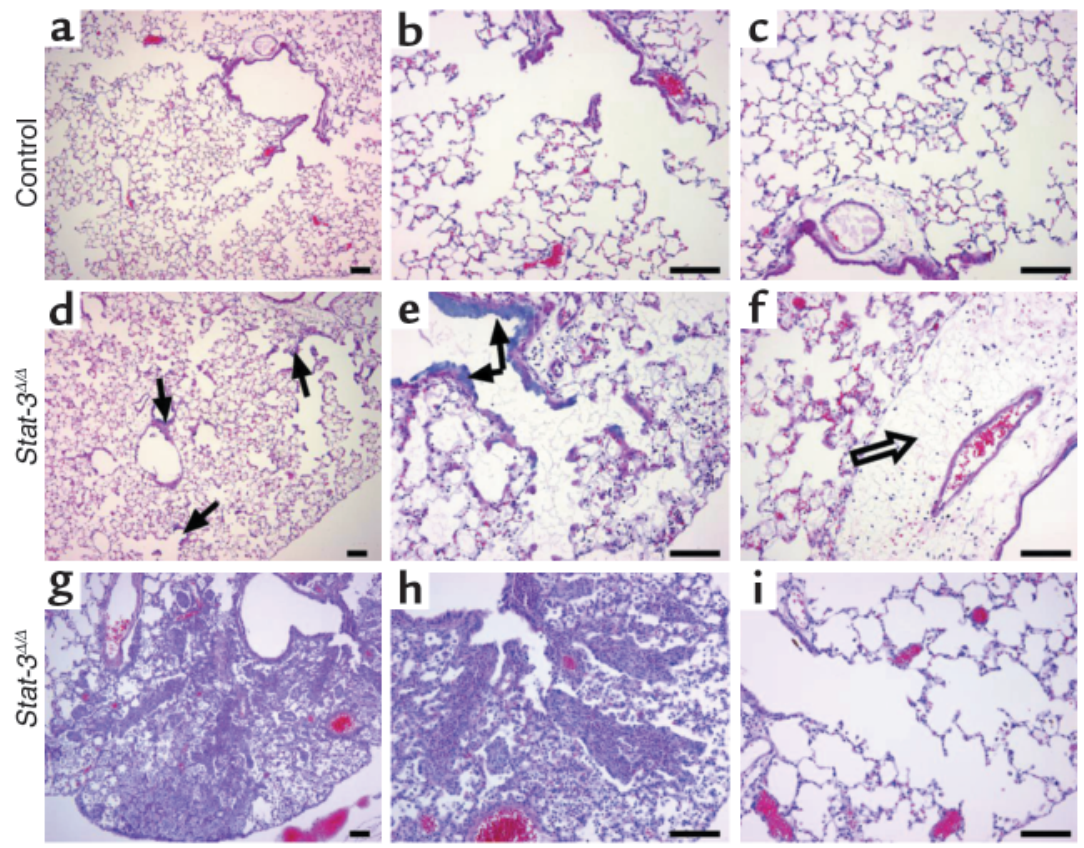
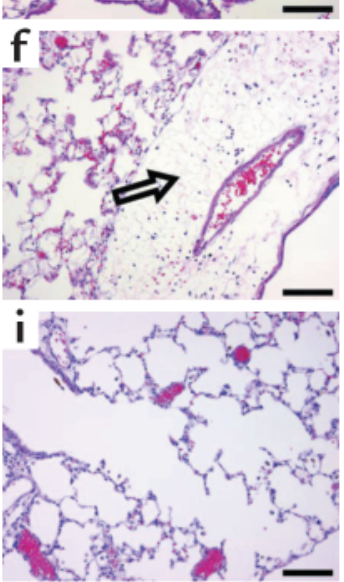

Figure 4

Lung morphology after exposure to $95 \% \mathrm{FiO}_{2}$. Adult (8-10 weeks of age) Stat- $3^{\Delta / \Delta}$ mice and nondeleted littermates were exposed to $95 \%$ $\mathrm{FiO}_{2}$ for 65 hours. Minor histologic changes (perivascular edema) in lung histology were observed in control mice after oxygen exposure (a-c). Severe epithelial cell necrosis (arrows), thickened alveoli, lymphotic edema (open arrow), and extensive inflammation were observed in the Stat-3 $3^{\Delta / \Delta}$ mice $(\mathbf{d}-\mathbf{f})$. Airspace enlargement was observed in some Stat-3 ${ }^{\Delta / \Delta}$ mice (g-i). Photomicrographs are representative of $n=7$ in each group. Scale bar: $100 \mu \mathrm{m}$. 
Table 1

Comparison of histopathology in oxygen-exposed control and Stat- $3^{\Delta / \Delta}$ mice

\begin{tabular}{|c|c|c|c|}
\hline Histopathologic features ${ }^{\mathrm{A}}$ & Controls ${ }^{B}$ & Stat- $3^{\Delta / \Delta B}$ & Significance ${ }^{C}$ \\
\hline Alveolar capillary membrane & Median & Median & $P$ value \\
\hline Congestion & 1 & 2 & $<0.001$ \\
\hline Hemorrhage & 0 & 1 & $<0.001$ \\
\hline Serum protein leak & 1 & 2.5 & $<0.001$ \\
\hline Hyaline membranes & 1 & 3 & $<0.001$ \\
\hline Septal wall edema & 0 & 2 & 0.005 \\
\hline Cellular necrosis & 1 & 3 & 0.011 \\
\hline Atelectasis & 0 & 0 & 0.575 \\
\hline \multicolumn{4}{|l|}{ Bronchiolar epithelia } \\
\hline Epithelial erosion & 1 & 4 & $<0.001$ \\
\hline Cellular necrosis & 0 & 4 & $<0.001$ \\
\hline Hyaline membranes & 0 & 4 & $<0.001$ \\
\hline Perivascular edema & 1 & 4 & $<0.001$ \\
\hline \multicolumn{4}{|l|}{ Inflammation } \\
\hline Cellularity & 0 & 4 & $<0.001$ \\
\hline Neutrophil infiltrates & 0 & 3 & $<0.001$ \\
\hline Macrophage infiltrates & 0 & 2 & $<0.001$ \\
\hline \multicolumn{4}{|l|}{ Remodeling } \\
\hline Alveolar enlargement & 0 & 0.5 & 0.039 \\
\hline General fibroproliferation & 0 & 0 & 0.886 \\
\hline Consolidation/fibrotic foci & 0 & 0 & 0.383 \\
\hline
\end{tabular}

AFor each feature, every lobe was scored from 0 to 5 , with 0 being normal or no histopathology, and 5 being the most severely affected. ${ }_{n} n=6$ mice from each group; $n=16$ lobes for control mice; $n=26$ lobes for Stat-3 ${ }^{4 / \Delta}$ mice. ${ }^{C}$ The median score was determined for each feature, and the Mann-Whitney rank sums test was performed to determine significant differences.

body weight of SP-B/DPPC/POPG or DPPC/POPG, and returned to $95 \%$ oxygen chamber.

Body weight. Body weights were similar for all groups with a mean of $24.9 \pm 1.2 \mathrm{~g}$ for control (double-transgenic littermates) and $25.3 \pm 1.1 \mathrm{~g}(n=6$ per group) for Stat- $3^{\Delta / \Delta}$ mice. Body weight losses were similar in all groups after 65 hours of exposure to hyperoxia $(16.7 \% \pm 2.4 \%$ versus $15.8 \% \pm 0.7 \%$ in control and Stat $-3 \Delta / \Delta$, respectively). Results were normalized to body weight by using body weight before oxygen exposure, since body weight correlates well with lung size (25).

Statistical analysis. Two group comparisons were carried out by unpaired Student's $t$ tests. Comparisons among groups were by ANOVA with Tukey's tests used for post hoc analyses. Z-test and log-rank test were used for percentage of survival comparison between groups. Results were expressed as mean plus or minus SE. Significance was accepted at the $5 \%$ level.

\section{Results}

Cell-specific deletion of Stat-3 in the respiratory epithelium. SP-

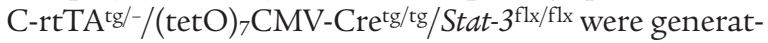
ed to produce Stat-3 $3^{\Delta / \Delta}$ mice in which Stat-3 is permanently deleted from intrapulmonary respiratory epithelial cells after administration of doxycycline to the dam (Figure 1). In this system, nearly complete gene recombination occurs in lung epithelial progenitor cells before E10.5 (13). The resulting mutation in the Stat-3 locus deletes exons 20-22 (14), producing Stat- $3^{\Delta / \Delta}$ mice
(Figure 1a). To document the deletion of STAT-3, type II alveolar epithelial cells were isolated from adult Stat$3^{\Delta / \Delta}$ and control mice, and STAT- 3 mRNA was assessed. Viability and purity of type II cells were not altered. STAT-3 mRNA was markedly decreased in cells from the Stat $-3^{\Delta / \Delta}$ mice (Figure $1 \mathrm{~b}$ ) to less than $10 \%$ of that in control cells. In wild-type, single, and double-transgenic littermates, immunohistochemical staining for STAT-3 was detected primarily in epithelial cells in both conducting airways and in type II cells in the alveoli, but was also detected in alveolar macrophages and other pulmonary cells. Virtually complete loss of nuclear STAT-3 staining was observed in intrapulmonary respiratory epithelial cells at E18.5 and in the adult Stat-3 $3^{\Delta / \Delta}$ mice (Figure 2). As expected from the specificity of the SP-C promoter, STAT-3 staining in alveolar macrophages and nonepithelial cells, including those of the lung vasculature and stroma, was unaltered (Figure $2 \mathrm{~h}$ ). Deletion of Stat-3 did not alter lung size, morphology, or survival at E18.5 or after birth. Stat- $3^{\Delta / \Delta}$ mice have had normal longevity in the vivarium. Thus, no apparent abnormalities in lung structure or function were observed following deletion of Stat-3 in respiratory epithelial cells.

Stat-3 $3^{\Delta / \Delta}$ Mice are susceptible to oxygen-induced injury. Adult Stat $-3^{\Delta / \Delta}$ mice and littermates were placed in $95 \% \mathrm{O}_{2}$. Initial studies indicated that $S t a t-3 \Delta / \Delta$ mice were more susceptible to hyperoxia as indicated by the time of onset of respiratory symptoms. Survival of Stat-3 $3^{\Delta / \Delta}$ mice in hyperoxia was significantly decreased (Figure 3). Consistent with this observation, the histopathology was consistent with increased severity of lung injury in the Stat- $3^{\Delta / \Delta}$ mice during hyperoxia (Table 1, Figure 4). Exposure to $95 \% \mathrm{O}_{2}$ for 65 hours caused mild congestion, severe protein leak, perivascular edema, and inflammation in Stat- $3^{\mathrm{fl} / \mathrm{flx}}$ control mice (Figure 4, a-c). Cellular necrosis and hyaline membranes were observed in the epithelium lining bronchiolar and alveolar regions of the lung. Hemorrhage, perivascular, and lymphatic edema, and loss of bronchi-

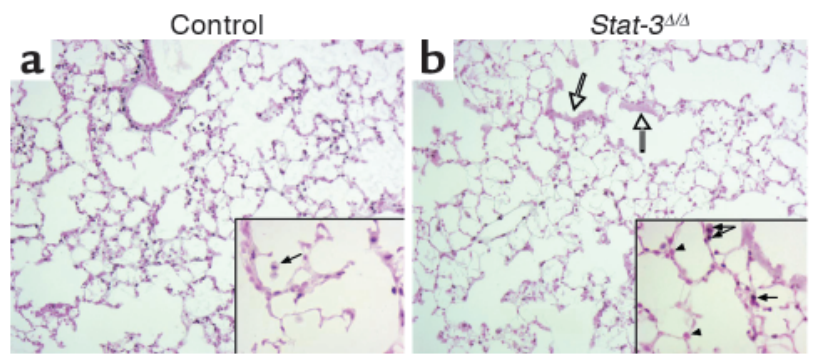

Figure 5

STAT-3 immunohistochemistry after exposure to $95 \%$ oxygen. Lungs from control (a) and Stat- $3^{\Delta / \Delta}$ (b) mice were stained for Stat-3 after 72 hours of exposure to $95 \% \mathrm{O}_{2}$. Alveolar type II cells, bronchiolar epithelial cells, and alveolar macrophages were stained in controls. Stat-3 was detected in inflammatory cells (filled arrows), vascular and stromal cells, but not in epithelial cells (arrowheads), in Stat- $3^{\Delta / \Delta}$ mice. Bronchiolar epithelial cell necrosis was prominent (open arrows). Original magnification $\times 10$. Magnification for inserted enlarged photomicrographs $\times 40$. 
a

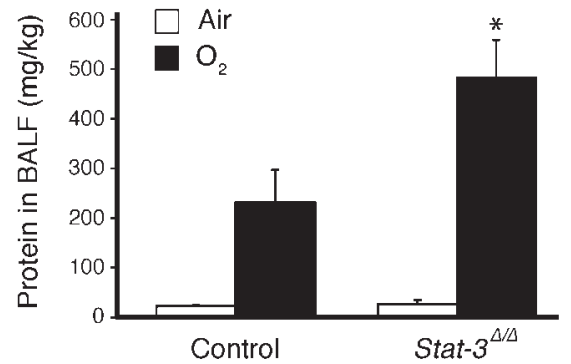

b

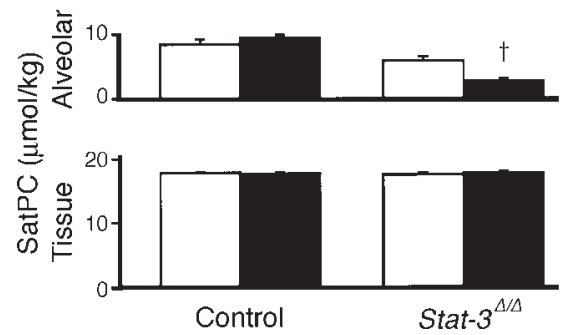

\section{Figure 6}

Increased alveolar capillary leak and decreased surfactant in Stat- $3^{\Delta / \Delta}$ mice after hyperoxia. (a) Total protein concentration was measured in lung lavage fluid 65 hours after exposure to $95 \% \mathrm{O}_{2}$. Protein content was similar in control and Stat-3 $3^{\Delta / \Delta}$ mice in room air. After oxygen exposure, BALF protein recovered from each mouse (micrograms per kilogram) was significantly increased in the Stat- $3^{\Delta / \Delta}$ mice, $n=5$ per group. ${ }^{*} P<0.05$ versus others and $P<0.001$ versus room air, as assessed by ANOVA. (b) Surfactant saturated phosphatidylcholine (SatPC) was significantly decreased in BALF from the Stat- $3^{\Delta / \Delta}$ mice following hyperoxia. Statistical differences were analyzed by ANOVA. $+P<0.001$ versus air.

olar and alveolar epithelia, were detected after oxygen exposure of Stat-3 $3^{\Delta / \Delta}$ mice, indicating severe lung injury (Figure 4, d-i). Epithelial and vascular cell necrosis, increased numbers of inflammatory cells, and accumulation of extracellular debris and protein were frequently observed in the $S t a t-3 \Delta / \Delta$ mice after exposure to oxygen. STAT-3 immunohistochemistry was performed after exposure to $95 \% \mathrm{O}_{2}$, demonstrating intense nuclear staining of inflammatory cells in both control and Stat- $3^{\Delta / \Delta}$ mice. STAT-3 immunostaining was not detected in most epithelial cells in peripheral airways or in the alveoli of Stat- $3^{\Delta / \Delta}$ mice (Figure 5), but was maintained in nonepithelial cells, including alveolar macrophages and pulmonary vascular-stromal cells.

Alveolar capillary leak and pulmonary dysfunction in Stat- $3^{\Delta / \Delta}$ mice. Protein content of lung lavage fluid was significantly increased and alveolar surfactant phospholipid (saturated phosphatidylcholine) pool sizes were significantly decreased, consistent with the increased alveolar capillary leak and decreased pulmonary surfactant pool size (Figure 6, a and b). While pulmonary mechanics were unaltered in $S t a t-3 \Delta / \Delta$ mice under normoxic conditions, exposure to $95 \% \mathrm{O}_{2}$ for 65 hours resulted in a marked decline in pulmonary function. Lung compliance was decreased in association with a marked increase in airway resistance, tissue damping, and elastance, consistent with severe surfactant dysfunction and tissue edema in Stat-3 $3^{\Delta / \Delta}$ mice (Figure 7). No differences were detected in hysteresivity and newtonian resistance.

Surfactant protein deficiency in Stat-3 $3^{4 / \Delta}$ mice. SP-B was undetectable in alveolar lavage of Stat-3 $3^{\Delta / \Delta}$ mice after 65 hours of oxygen exposure (Figure 8). Likewise, SP-A was significantly decreased in $S t a t-3^{\Delta / \Delta}$ mice after hyperoxia. Immunostaining demonstrated a marked reduction in SP-B in both bronchiolar and alveolar epithelial cells in the oxygen-treated Stat- $3^{\Delta / \Delta}$ mice (Figure 9). Staining for proSP-C, a selective marker for type II cells, was present, but was also decreased (Figure 8). Consistent with these observations, surfactant protein mRNAs (SP-A, SP-B, and $\mathrm{SP}-\mathrm{C}$ ) were decreased after oxygen exposure, and the level of SP-B mRNA was significantly reduced in Stat- $3^{\Delta / \Delta}$ compared with control after 65 hours of $95 \%$ $\mathrm{O}_{2}$ (Figure 8b). Since reduction of alveolar SP-B to less than $50 \%$ of normal renders mice susceptible to respiratory failure during hyperoxia, we considered whether the loss of SP-B may influence susceptibility of the Stat $-3^{\Delta / \Delta}$ mice to oxygen.

Exogenous $S P-B$ protects Stat-3 $3^{\Delta / \Delta}$ mice during byperoxia. Stat $-3^{\Delta / \Delta}$ mice were treated intratracheally with a synthetic exogenous surfactant containing $4 \%$ human SP-B and lipids or lipids alone ( $n=8$ per group) 16 and 40 hours after initiation of $95 \%$ oxygen. The experiment was not continued after 5 days in hyperoxia because all surviving mice had developed severe respiratory distress. Treatment of the $S t a t-3 \Delta / \Delta$ mice with SP-B/DPPC/POPG mixture significantly improved survival and lung histology 4 days after exposure to oxygen (Figure 10).
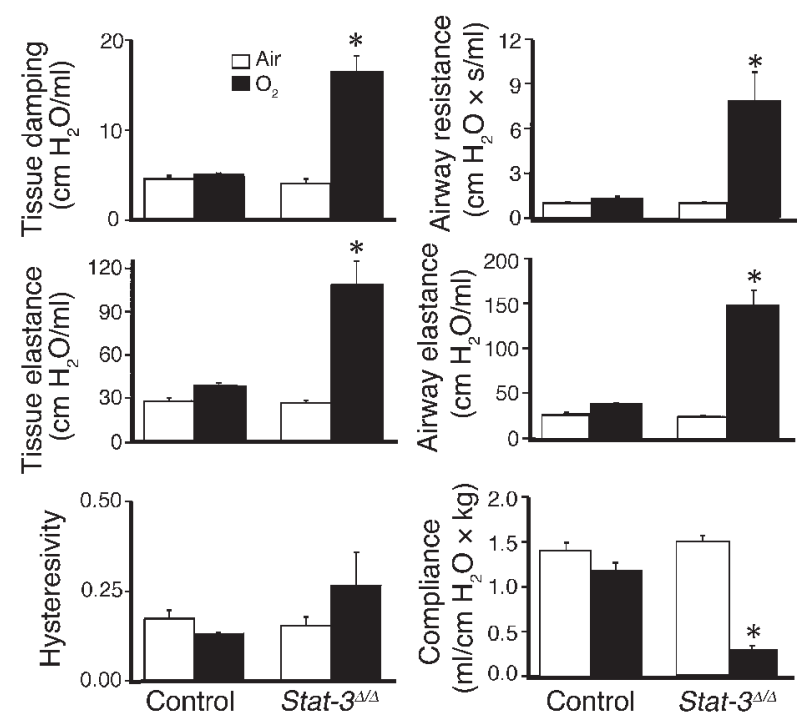

Figure 7

Abnormalities in pulmonary mechanics in Stat-3 $3^{\Delta / \Delta}$ mice following hyperoxia. Lung mechanics were assessed in adult control and Stat- $3^{\Delta / \Delta}$ mice exposed to room air or $95 \% \mathrm{O}_{2}$ for 65 hours. Lung mechanics were similar in control and Stat-3 $3^{\Delta / \Delta}$ mice in room air. After exposure to $95 \% \mathrm{O}_{2}$, airway resistance, airway elastance, tissue damping, and tissue elastance were increased and compliance decreased significantly in Stat- $3^{\Delta / \Delta}$ but not in control mice. $n=5$ per group. Statistical differences were analyzed by ANOVA. ${ }^{*} P<0.01$ versus control group. 
a
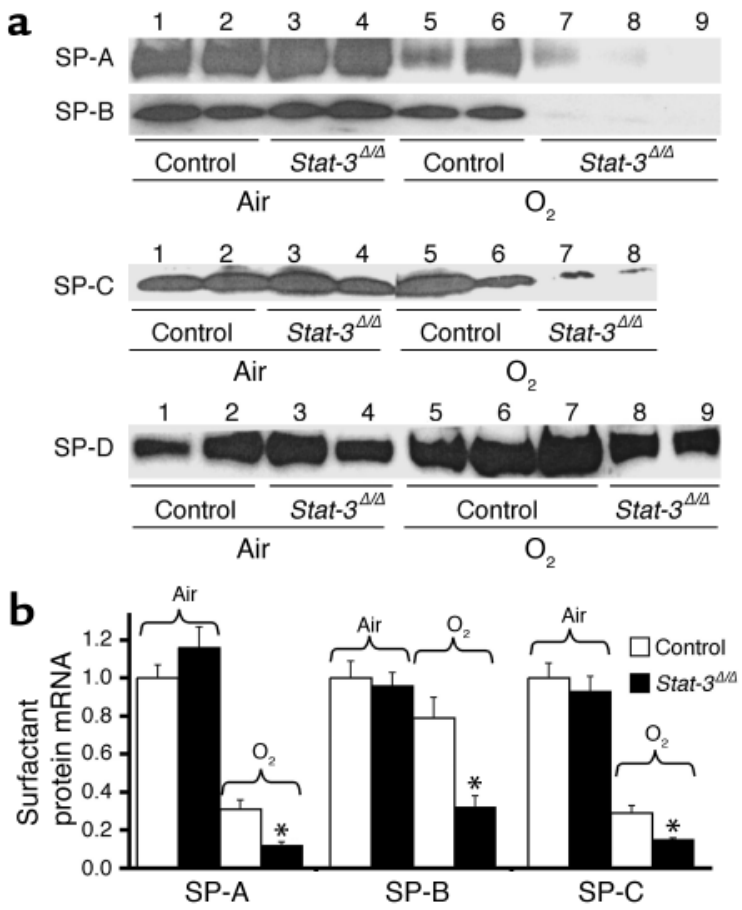

Inflammation and cellular injury in Stat-3 $3^{\Delta / \Delta}$ mice after oxygen exposure. Epithelial sloughing, hyaline membrane formation, loss of alveolar capillary membrane integrity, and increased inflammation were consistent with severe epithelial cell injury in the Stat- $3^{\Delta / \Delta}$ mice. Increased infiltration by polymorphonuclear cells and alveolar macrophages was observed in association with an increased production of IL- $1 \beta$, IL-6, and MIP- 2 in the Stat- $3^{\Delta / \Delta}$ mice following hyperoxia (Figure 11). While histologic evaluation demonstrated severe epithelial and vascular cell necrosis, no DNA laddering was observed. Caspase-3, keratin-18 fragments, and TUNEL staining identified only rare positive cells, usually found in isolated clusters in conducting airways (data not shown). Thus, widespread epithelial cell necrosis was readily apparent after oxygen exposure of Stat-3 $3^{\Delta / \Delta}$ mice without evidence of apoptosis.

\section{Discussion}

While respiratory epithelial cell-specific deletion of Stat-3 did not alter lung morphogenesis or function, STAT- 3 was required for maintenance of alveolar capillary integrity, surfactant homeostasis, and normal

\footnotetext{
Figure 9

Decreased immunostaining for SP-B and proSP-C in Stat- $3^{\Delta / \Delta}$ mice after exposure to $95 \% \mathrm{FiO}_{2}$. Staining for SP-B (a-d) and proSP-C (e-h) was performed in Stat-3 $3^{\Delta / \Delta}$ and control littermates exposed to room air or $95 \% \mathrm{O}_{2}$. In room air there were no differences in intensity or distribution of SP-B and proSP-C staining between Stat-3 $3^{\Delta / \Delta}$ and control mice. After 65 hours in 95\% oxygen, SP-B and proSP-C staining were decreased in Stat-3 $3^{\Delta / \Delta}$ ( $\mathbf{d}$ and $\mathbf{h}$ ) compared with controls ( $\mathbf{b}$ and $\mathbf{f}$ ). Scale bar: $100 \mu \mathrm{m}$. Photomicrographs are representative of $n \geq 3$.
}

\section{Figure 8}

Decreased surfactant proteins in Stat- $3^{\Delta / \Delta}$ mice following hyperoxia. (a) SP-A, SP-B, SP-C, and SP-D were quantitated in BAL fluid by Western blot analysis. In room air, SP-A, SP-B, SP-C, and SP-D were not altered (lanes 3 and 4). After exposure to $95 \% \mathrm{O}_{2}$ for 65 hours, SP-A and SP-B were significantly decreased in the Stat- $3^{\Delta / \Delta}$ mice (lanes 7-9), $P<0.01$, after quantitative imaging. (b) SP-A, SP-B, and SP-C mRNAs were quantitated by $\mathrm{S} 1$ nuclease assay. In room air, SP-A, SP-B, and SP-C mRNAs were similar in Stat- $3^{\Delta / \Delta}$ and control mice. After exposure to $95 \% \mathrm{O}_{2}$ for 65 hours, SP-A, SP-B, and SP-C mRNAs were decreased in Stat- $3^{\Delta / \Delta}$ compared with control mice. Results were standardized to L32 mRNA and normalized to room air controls, $n=5$ per group. Statistical differences were assessed by Student's $t$ test, ${ }^{*} P<0.01$ versus control $\mathrm{O}_{2}$.

pulmonary mechanics during oxygen-induced lung injury. In the absence of STAT-3, hyperoxia caused ALI with epithelial and vascular injury, leading to respiratory compromise. Loss of SP-B and surfactant lipids and increased alveolar capillary leak were associated with deterioration of pulmonary mechanics. Intratracheal SP-B significantly improved lung histology and survival of Stat-3 $3^{\Delta / \Delta}$ mice during hyperoxia.

Conditional targeting of STAT-3 in the lung. STAT-3 is widely expressed in the developing embryo, and its
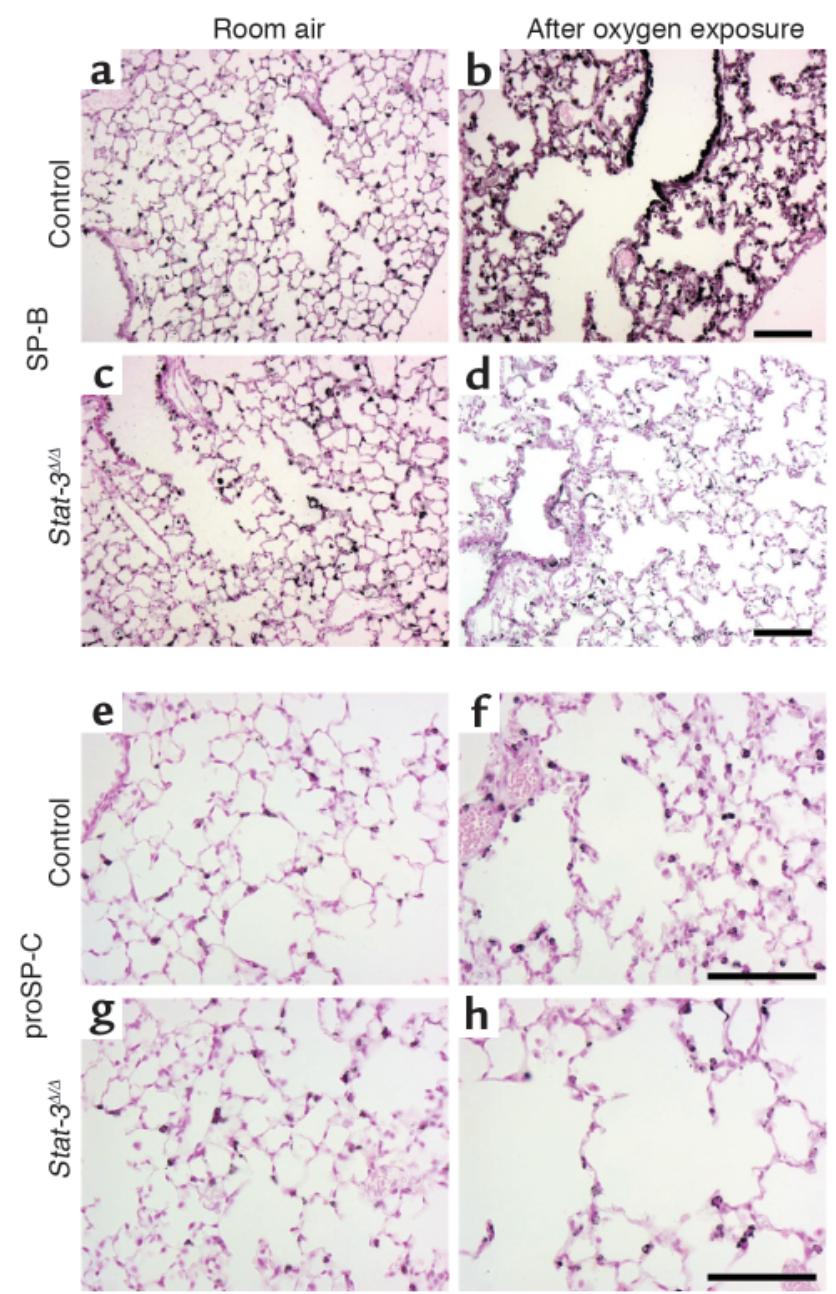

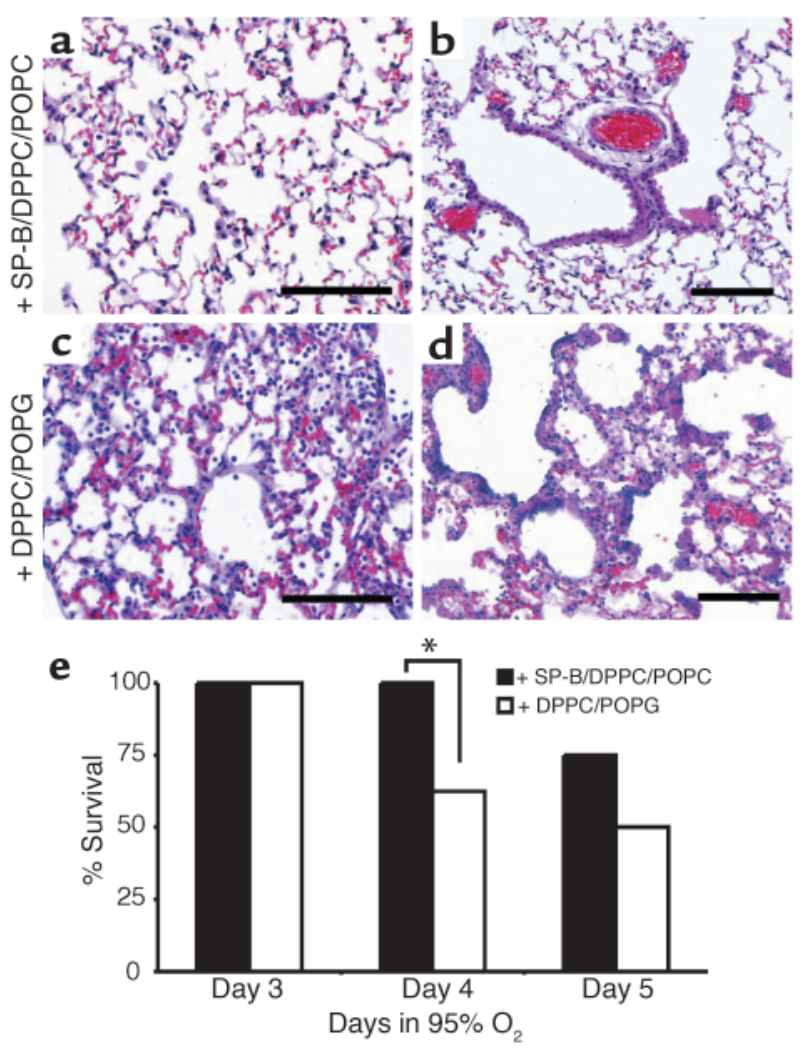

deletion is lethal by E6-7 in mice, well before the formation of the respiratory tract. The lung buds are formed at approximately E9-9.5 from cells at the lateral tracheal-esophageal sulcus in the foregut endoderm. In the present studies, exposure of the fetus to doxycycline-induced expression of Cre-recombinase in pulmonary progenitor cells prior to lung bud formation results in extensive recombination of intrapulmonary respiratory epithelial cells and their daughter cells, causing nearly complete deletion of STAT-3 in bronchiolar and alveolar epithelial cells within the lung (13). Decreased immunostaining for STAT-3 was observed in epithelial cells in the lungs of the Stat- $3^{\Delta / \Delta}$ mice. The specificity and extent of deletion was further confirmed by the marked decrease in STAT- 3 mRNA in isolated type II cells from the Stat-3 $3^{\Delta / \Delta}$ mice. In this system, gene targeting occurs in progenitor cells that contribute to virtually all epithelial cell types in the peripheral lung except neuroepithelial cells (13). As expected from the specificity of the SP-C promoter, Stat-3 gene expression was deleted in pulmonary epithelial cells but not in alveolar macrophages, vascular cells, or other pulmonary cells. Remarkably, loss of STAT-3 did not perturb lung morphogenesis or surfactant homeostasis after birth. Thus, STAT-3 is not required for lung formation or function in the absence of injury. These findings are consistent with those in which the cytokeratin K5-Cre transgenic mice were used to delete STAT-3 in epithelial cells of the skin (26). In those studies, neonatal skin formation and proliferation were not perturbed; however, hair follicle

\section{Figure 10}

Exogenous SP-B protects Stat- $3^{\Delta / \Delta}$ mice during hyperoxia. (a) Lung morphology of the Stat-3 $3^{\Delta / \Delta}$ mice treated with SP-B/DPPC/POPG (a and $\mathbf{b}$ ) or DPPC/POPG (c and $\mathbf{d}$ ) during hyperoxia exposure is shown. Lungs were excised 65 hours after exposure to $95 \%$ oxygen and stained with H\&E. The lungs of SP-B/DPPC/DOPG-treated mice had less inflammatory cell infiltration, and epithelial cell necrosis was not observed ( $\mathbf{a}$ and $\mathbf{b}$ ). Alveolar thickening, inflammation, and epithelial cell necrosis was widespread in mice treated with DPPC/DOPG alone (c and $\mathbf{d}$ ). Photomicrographs are representative of $n=5$ from each group. Bars $=100 \mu \mathrm{m}$. (e) SP-B enhances survival of Stat- $3^{\Delta / \Delta}$ mice during hyperoxia. Stat- $3^{\Delta / \Delta}$ mice were placed in $95 \%$ oxygen and treated (intratracheally) with SP-B/DPPC/DOPG or DPPC/DOPG as described in Methods. Survival on day 4 was significantly increased in SP-B/DPPC/DOPG-treated mice; ${ }^{*} P<0.05$. On day 5, more SP-B/DPPC/DOPG-treated mice survived, but differences were not statistically significant; $n=8$ per group.

maturation and wound healing was markedly impaired in the STAT-3-deficient mice.

STAT-3 is required for maintenance of lung function during hyperoxia. Continued exposure to hyperoxia causes progressive lung injury with alveolar capillary leak and respiratory failure $(27,28)$. The time course and pathogenesis of lung injury following oxygen exposure in the mouse are well characterized. Consistent with previous findings, Stat- $3 \mathrm{fl} / \mathrm{fl} x \mathrm{x}$ control mice generally tolerated $95 \%$ oxygen for 3-4 days, developing severe respiratory symptoms after 5 or more days of continued exposure (28). In contrast, Stat-3 $3^{\Delta / \Delta}$ mice developed acute respiratory distress within 72 hours of hyperoxia. The changes in lung structure, lung permeability, and surfactant content in Stat- $3^{\Delta / \Delta}$ mice caused by hyperoxia were associated with abnormalities in lung mechanics. Epithelial cell necrosis was extensive in the bronchiolar epithelium of the Stat- $3^{\Delta / \Delta}$ mice after oxygen exposure, a finding commonly seen in respiratory distress syndromes (RDSs) in preterm infants and in ARDS in adults. The observed epithe-

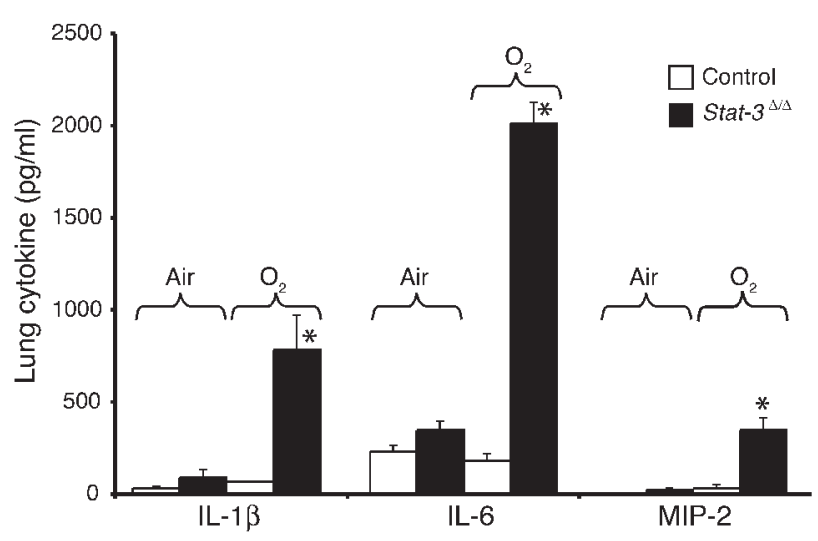

\section{Figure 11}

Increased IL-1 $\beta$, IL-6, and MIP-2 in Stat-3 $3^{\Delta / \Delta}$ mice after $95 \% \mathrm{O}_{2}$. IL-1 $\beta$, $\mathrm{IL}-6$, and MIP-2 were measured in lung tissue from control and Stat- $3^{\Delta / \Delta}$ mice exposed to room air or $95 \% \mathrm{O}_{2}$ for 65 hours. Levels of each cytokine were significantly increased in the Stat- $3^{\Delta / \Delta}$ mice after hyperoxia, $n=5$ per group, as determined by ANOVA; ${ }^{*} P<0.001$ versus others. 
lial cell injury in both bronchiolar and alveolar regions of the lung is consistent with the sites of gene targeting in this transgenic model.

In the present study, SP-B mRNA and SP-B content was markedly reduced after exposure of the $S t a t-3^{\Delta / \Delta}$ mice to $95 \%$ oxygen. Gene targeting or mutations of $S f t p B$, but not $S f t p A$ and $S f t p C$, cause lethal respiratory failure at birth, demonstrating an absolute requirement for SP-B for lung function in the postnatal period $(10,29,30)$. Even heterozygous $S f t p B^{-/-}$mice, in which SP-B mRNA and protein are reduced $50 \%$, were susceptible to oxygen-induced lung injury in vivo (31), and increased expression of SP-B following oxygen exposure is required for maintenance of lung function (19). Taken together with the findings that intratracheal SP-B enhanced survival and improved lung histology, the susceptibility of the Stat-3 $3^{\Delta / \Delta}$ mice to hyperoxia is, at least in part, mediated by its effect on maintenance of SP-B. The severity of lung injury and loss of bronchiolar and alveolar cell integrity seen in the Stat $-3^{\Delta / \Delta}$ mice is not seen in SP-B-deficient mice (31, 32 ), suggesting that STAT-3 is likely to mediate protective or repair responses independently of the loss of SP-B. The present findings are consistent with previous in vitro observations that STAT-3 directly activates $S f t p B$ gene transcription (6), but also demonstrate that STAT-3 is not required for SP-B production and surfactant function under normal physiologic conditions. STAT- 3 is activated by a number of cytokines of the IL-6 family of cytokines (including IL-6, IL-11, leukemia inhibitory factor, and others) and non-IL-6 cytokines (IL-2, IL-5, IL-7, IL-10, IFN- $\alpha$, and IFN- $\gamma$ ). Growth factors including HGF, EGF, TGF- $\alpha$, human epidermal growth factor receptor-2 (HER2/neu), PDGF, G-CSF, and leptin are also known to activate STAT-3 in various cell types (33; see ref. 5 for review). In the present study, increased inflammation was associated with increased production of the inflammatory mediators IL-6, IL-1 $\beta$, and MIP-2, perhaps indicating increased lung injury or a response related to the lack of STAT-3. Previous studies support cytoprotective effects of several of these ligands during lung injury. Of particular relevance to the present work, transgenic mice expressing increased amounts of TGF- $\alpha$, IL-11, or IL- 6 were found to be resistant to oxidant-induced lung injury (34-36). In in vitro studies, IL-6 and IL-11 protected endothelial cells from oxidant injury, and this protection is, at least in part, by a STAT-3-dependent pathway (37). In the present study, it is unclear which of the potential ligand and receptor pathways mediated STAT-3 functions are required for adaptation and survival of oxygen injury. Cell proliferation was difficult to assess in Stat- $3^{\Delta / \Delta}$ mice during hyperoxia. BrdU or proliferating cell nuclear antigen-stained cells were relatively rare, and the mice did not survive. Since Stat-3 $3^{\Delta / \Delta}$ mice developed respiratory failure after relatively short periods of hyperoxia, the potential role of STAT- 3 in the repair process could not be readily assessed in the present model. While widespread cellular injury was observed in the Stat-3 $3^{\Delta / \Delta}$ mice during hyperoxia, neither DNA laddering, TUNEL, nor caspase-3 staining indicated that apoptosis played a primary role in their susceptibility to oxygen injury.

Clinically, RDSs in preterm infants and adults is caused by or associated with surfactant deficiency. Replacement of pulmonary surfactant at birth in preterm infants with RDSs is associated with protection of the lung epithelium and maintenance of lung function. Surfactant dysfunction and the loss of SP-B has been observed in association with ARDS in humans $(8,38)$, although many factors, including vascular-endothelial cell injury and inflammation, may contribute to the pathogenesis of respiratory failure. Oxygen and ventilation therapy, required for maintenance of lung function in critically ill patients, may further contribute to epithelial and vascular cell injury. Since alveolar SP-B was markedly decreased or absent in the Stat-3 $3^{\Delta / \Delta}$ mice after oxygen injury, the loss of SP-B alone is sufficient to explain the observed respiratory failure in these mice. Consistent with this concept, conditional reduction of SP-B to $20-30 \%$ of control levels in transgenic mice causes respiratory distress and death in adult mice, but does not cause the widespread cellular injury presently seen in the Stat-3 $3^{\Delta / \Delta}$ mice during hyperoxia. Likewise, loss of SP-B related to mutations in the $S f t p B$ gene caused surfactant abnormalities and respiratory failure at birth but did not directly cause epithelial cell necrosis $(9,10)$. Exogenous SP-B enhanced lung function and survival of the Stat- $3^{\Delta / \Delta}$ mice in hyperoxia, delaying but not blocking respiratory failure in this model.

The present study demonstrates a critical requirement for STAT-3 in respiratory epithelial cells during exposure to $95 \%$ oxygen. The rapid onset of respiratory dysfunction and increased severity of epithelial cell injury and alveolar capillary leak provides support for the concept that STAT-3 influences expression of surfactant proteins, as well as other processes required for maintenance of lung function during hyperoxia. From a clinical perspective, activation of pathways maintaining or increasing Stat-3 and surfactant protein B expression in respiratory epithelial cells may provide a strategy for protection of the lung during acute or chronic injury associated with ALI syndromes.

\section{Acknowledgments}

This work was supported by NIH grants HL61646 (M. Ikegami, S.E. Wert, J.A. Whitsett) and HL38859 (J.A. Whitsett).

\footnotetext{
1. Akira, S. 1997. IL-6-regulated transcription factors. Int. J. Biochem. Cell Biol. 29:1401-1418.

2. Darnell, J.E., Jr. 1997. STATs and gene regulation. Science. 277:1630-1635.

3. Boccaccio, C., et al. 1998. Induction of epithelial tubules by growth factor HGF depends on the STAT pathway. Nature. 391:285-288.

4. Takeda, K., et al. 1997. Targeted disruption of the mouse Stat 3 gene leads to early embryonic lethality. Proc. Natl. Acad. Sci. U. S. A. 94:3801-3804.

5. Levy, D.E., and Lee, C.K. 2002. What does Stat3 do? J. Clin. Invest. 109:1143-1148. doi:10.1172/JCI200215650.

6. Yan, C., et al. 2002. Transcriptional stimulation of the surfactant pro-
} 
tein B gene by STAT3 in respiratory epithelial cells. J. Biol. Chem. 277:10967-10972.

7. Riley, S., et al. 2003. Transmission dynamics of the aetiological agent of severe acute respiratory syndrome (SARS) in Hong Kong: The impact of public health interventions. Science. 300:1961-1966.

8. Gregory, T.J., et al. 1991. Surfactant chemical composition and biophysical activity in acute respiratory distress syndrome. J. Clin. Invest. 88:1976-1981.

9. Poulain, F.R., Allen, L., Williams, M.C., Hamilton, R.L., and Hawgood, S. 1992. Effects of surfactant apolipoproteins on liposome structure: implications for tubular myelin formation. Am. J. Physiol. 262:L730-L739.

10. Clark, J.C., et al. 1995. Targeted disruption of the surfactant protein B gene disrupts surfactant homeostasis, causing respiratory failure in newborn mice. Proc. Natl. Acad. Sci. U. S. A. 92:7794-7798.

11. Nogee, L.M. 1997. Surfactant protein-B deficiency. Chest. 111(Suppl. 6):129S-135S

12. Tichelaar, J.W., Lu, W., and Whitsett, J.A. 2000. Conditional expression of fibroblast growth factor-7 in the developing and mature lung. J. Biol. Chem. 275:11858-11864.

13. Perl, A.K., Wert, S.E., Nagy, A., Lobe, C.G., and Whitsett, J.A. 2002. Early restriction of peripheral and proximal cell lineages during formation of the lung. Proc. Natl. Acad. Sci. U. S. A. 99:10482-10487.

14. Takeda, K., et al. 1998. Stat3 activation is responsible for IL-6-dependent $\mathrm{T}$ cell proliferation through preventing apoptosis: generation and characterization of T cell-specific Stat3-deficient mice. J. Immunol. 161:4652-4660

15. Rice, W.R., et al. 2002. Maintenance of the mouse type II cell phenotype in vitro. Am. J. Physiol. 283:L256-L264.

16. Shi, S.R., Key, M.E., and Kalra, K.L. 1991. Antigen retrieval in formalinfixed, paraffin-embedded tissues: an enhancement method for immunohistochemical staining based on microwave oven heating of tissue sections. J. Histochem. Cytochem. 39:741-748.

17. Zhou, L., Lim, L., Costa, R.H., and Whitsett, J.A. 1996. Thyroid transcription factor-1, hepatocyte nuclear factor-3beta, surfactant protein $\mathrm{B}, \mathrm{C}$, and Clara cell secretory protein in developing mouse lung. J. Histochem. Cytochem. 44:1183-1193.

18. Dranoff, G., et al. 1994. Involvement of granulocyte-macrophage colony stimulating factor in normal pulmonary homeostasis. Science. 264:713-716.

19. Tokieda, K., et al. 1999. Surfactant protein B corrects oxygen-induced pulmonary dysfunction in heterozygous surfactant protein B-deficient mice. Pediatr. Res. 46:708-714.

20. Schuessler, T.F., and Bates, J.H. 1995. A computer-controlled research ventilator for small animals: design and evaluation. IEEE Trans. Biomed. Eng. 42:860-866.
21. Hantos, Z., Daroczy, B., Suki, B., Nagy, S., and Fredberg, J.J. 1992. Input impedance and peripheral inhomogeneity of dog lungs. J. Appl. Physiol. 72:168-178.

22. Lowry, O.H., Rosebrough, N.J., Farr, A.L., and Randall, R.J. 1951. Protein measurement with the Folin phenol reagent J. Biol. Chem. 193:265-275.

23. Mason, R.J., Nellenbogen, J., and Clements, J.A. 1976. Isolation of disaturated phosphatidylcholine with osmium tetroxide. J. Lipid. Res 17:281-284.

24. Bartlett, G.R. 1959. Phosphorus assay in column chromatography. J. Biol. Chem. 234:466-468.

25. Ohashi, T., Pinkerton, K., Ikegami, M., and Jobe, A.H. 1994. Changes in alveolar surface area, surfactant protein A, and saturated phosphatidylcholine with postnatal rat lung growth. Pediatr. Res. 35:685-689.

26. Sano, S., et al. 1999. Keratinocyte-specific ablation of Stat3 exhibits impaired skin remodeling, but does not affect skin morphogenesis. EMBO J. 18:4657-4668.

27. Smith, L.J., Frank, L., and Massaro, D. 1980. Oxygen toxicity. Am. J. Med. 69:117-126.

28. Smith, L.J. 1985. Hyperoxic lung injury: biochemical, cellular, and morphologic characterization in the mouse. J. Lab. Clin. Med. 106:269-278.

29. Glasser, S.W., et al. 2001. Altered stability of pulmonary surfactant in SP-C-deficient mice. Proc. Natl. Acad. Sci. U. S. A. 98:6366-6371.

30. Korfhagen, T.R., et al. 1996. Altered surfactant function and structure in SP-A gene targeted mice. Proc. Natl. Acad. Sci. U. S. A. 93:9594-9599.

31. Tokieda, K., et al. 1999. Surfactant protein-B-deficient mice are susceptible to hyperoxic lung injury. Am. J. Respir. Cell Mol. Biol. 21:463-472.

32. Melton, K.R., et al. 2003. SP-B deficiency causes respiratory failure in adult mice. Am. J. Physiol. 285:L543-L549.

33. Akira, S. 2000. Roles of STAT3 defined by tissue-specific gene targeting. Oncogene. 19:2607-2611.

34. Ward, N.S., et al. 2000. Interleukin-6-induced protection in hyperoxic acute lung injury. Am. J. Respir. Cell. Mol. Biol. 22:535-542.

35. Waxman, A.B., et al. 1998. Targeted lung expression of interleukin-11 enhances murine tolerance of $100 \%$ oxygen and diminishes hyperoxiainduced DNA fragmentation. J. Clin. Invest. 101:1970-1982.

36. Hardie, W.D., Prows, D.R., Leikauf, G.D., and Korfhagen, T.R. 1999 Attenuation of acute lung injury in transgenic mice expressing human transforming growth factor-alpha. Am. J. Physiol. 277:L1045-L1050.

37. Waxman, A.B., et al. 2003. IL-11 and IL-6 protect cultured human endothelial cells from $\mathrm{H}_{2} \mathrm{O}_{2}$-induced cell death. Am. J. Respir. Cell Mol. Biol. 29:513-522.

38. Pison, U., et al. 1989. Surfactant abnormalities in patients with respiratory failure after multiple trauma. Am. Rev. Respir. Dis. 140:1033-1039. 